\title{
Analyticity of the Schrödinger propagator on the Heisenberg group
}

\author{
S. Parui · P. K. Ratnakumar · S. Thangavelu
}

Received: 17 May 2011 / Accepted: 22 June 2012

(C) Springer-Verlag 2012

\begin{abstract}
We discuss the analytic extension property of the Schrödinger propagator for the Heisenberg sublaplacian and some related operators. The result for the sublaplacian is proved by interpreting the sublaplacian as a direct integral of an one parameter family of dilated special Hermite operators.
\end{abstract}

Keywords Schrödinger equation - Oscillatory group - Special Hermite expansion · Heisenberg group $\cdot$ Sublaplacian

Mathematics Subject Classification (1991) Primary 22E30;

Secondary $35 \mathrm{G} 10 \cdot 47 \mathrm{~A} 63$

\section{Introduction}

It is well known that the heat equation is infinitely smoothing in the sense that the solution $u(x, t)$ of the heat equation $\partial_{t} u=\Delta u$ with initial condition $u(x, 0)=f(x)$

Communicated by K. Gröchenig.

S. Parui $(\bowtie)$

School of Mathematics, National Institute of Science Education and Research, IOP Campus, Bhubaneswar 751005, India

e-mail: parui@niser.ac.in

P. K. Ratnakumar

Harish-Chandra Research Institute, Chhatnag Road, Jhunsi, Allahabad 211019, India

e-mail: ratnapk@hri.res.in

S. Thangavelu

Department of Mathematics, Indian Institute of Science, Bangalore 560012, India

e-mail: veluma@math.iisc.ernet.in 
is real analytic even if $f$ is only in $L^{2}\left(\mathbb{R}^{n}\right)$. Actually much more is true: if $q_{t}$ stands for the heat kernel,

$$
q_{t}(x)=(4 \pi t)^{-\frac{n}{2}} e^{-\frac{1}{4 t}|x|^{2}}
$$

then for $f \in L^{2}\left(\mathbb{R}^{n}\right)$, the solution $u(x, t)=f * q_{t}(x)$ extends to $\mathbb{C}^{n}$ as an entire function $u(x+i y, t)$ which satisfies

$$
\int_{\mathbb{R}^{2 n}}|u(x+i y, t)|^{2} q_{t / 2}(y) d x d y=c_{n} \int_{\mathbb{R}^{n}}|f(x)|^{2} d x .
$$

Conversely, any entire function $F(x+i y)$ on $\mathbb{C}^{n}$ for which

$$
\int_{\mathbb{R}^{2 n}}|F(x+i y)|^{2} q_{t / 2}(y) d x d y<\infty
$$

can be factored as $F(x)=f * q_{t}(x)$ for some $f \in L^{2}\left(\mathbb{R}^{n}\right)$. This is a celebrated theorem attributed to Bargmann [1].

In contrast to this, the Schrödinger equation cannot be expected to have such a smoothness property. In fact, the solution $v(x, t)$ of the equation

$$
i \partial_{t} v=\Delta v, v(x, 0)=f(x)
$$

is given by the unitary group $e^{-i t \Delta}$, which is an isometry onto $L^{2}$, so without any improvement in the smoothing. In view of this, the following result of Hayashi and Saitoh [9] looks surprising at the first sight. In 1990, they have shown that the solution of the one dimensional Schrödinger equation

$$
i \partial_{t} u+\frac{1}{2} \partial_{x}^{2} u=0, u(x, 0)=f(x), t, x \in \mathbb{R}
$$

extends to $\mathbb{C}$ as an entire function, provided the initial condition $f$ has enough decay at infinity. More precisely, they have shown that when $f$ is square integrable with respect to $e^{x^{2}} d x$, then $u(x, t)$ extends to $\mathbb{C}$ as an entire function and

$$
\frac{1}{\sqrt{\pi t^{2}}} \int_{-\infty}^{\infty} \int_{-\infty}^{\infty} e^{-\frac{y^{2}}{t^{2}}}\left|e^{-\frac{i}{2 t} z^{2}} u(z, t)\right|^{2} d x d y=\int_{-\infty}^{\infty}|f(x)|^{2} e^{x^{2}} d x
$$

for all $t \neq 0$. Their proof is based on the identity

$$
\frac{1}{\sqrt{\pi \alpha}} \int_{-\infty}^{\infty} \int_{-\infty}^{\infty} e^{-\frac{y^{2}}{\alpha}}|f(x+i y)|^{2} d x d y=\sum_{j=0}^{\infty} \frac{\alpha^{j}}{j !} \int_{-\infty}^{\infty}\left|\partial_{x}^{j} f(x)\right|^{2} d x
$$


However, the result can be directly proved in all dimensions without any recourse to the above identity. Indeed, the solution of the Schrödinger equation

$$
i \partial_{t} u=\Delta u, u(x, 0)=f(x)
$$

is given by

$$
u(x, t)=(4 \pi i t)^{-\frac{n}{2}} \int_{\mathbb{R}^{n}} f(y) e^{-\frac{1}{4 i t}|x-y|^{2}} d y .
$$

Therefore, $u(x, t) e^{\frac{1}{4 i t}|x|^{2}}$ is up to a constant multiple, the Fourier transform (at $\frac{1}{2 t} x$ ) of the function $f(y) e^{-\frac{1}{4 i t}|y|^{2}}$. If we assume that $f$ has a Gaussian decay, then it is well known that its Fourier transform extends to $\mathbb{C}^{n}$ as a holomorphic function. Therefore, it is not surprising that the solution of the Schrödinger equation also has some smoothing properties, provided we start with initial conditions having enough decay.

If we assume that $f$ is square integrable with respect to $e^{|y|^{2}} d y$, then a simple calculation shows that

$$
u(x, t) e^{-\frac{i}{4 t} \sum x_{j}^{2}}=(4 \pi i t)^{-\frac{n}{2}} \int_{\mathbb{R}^{n}}\left(f(y) e^{\frac{i}{4 t} \sum y_{j}^{2}}\right) e^{-\frac{i}{2 t} x \cdot y} d y,
$$

which can be written as

$$
u(x, t) e^{-\frac{i}{4 t} \sum x_{j}^{2}}=(2 i t)^{-n / 2} \hat{g} * q_{s}\left(\frac{1}{2 t} x\right)
$$

where $g(y)=f(y) e^{\frac{i}{4 t}|y|^{2}} e^{s|y|^{2}}$. As $g \in L^{2}\left(\mathbb{R}^{n}\right)$, it follows from the theorem of Segal and Bargmann that $F(x, t)=u(x, t) e^{-\frac{i}{4 t} \sum x_{j}^{2}}$ extends to $\mathbb{C}^{n}$ as an entire function and

$$
\begin{aligned}
\int_{\mathbb{C}^{n}}|F(z, t)|^{2} q_{s / 2}\left(\frac{y}{2 t}\right) d x d y & =(2 t)^{n} \int_{\mathbb{C}^{n}}\left|\hat{g} * q_{s}(z)\right|^{2} q_{s / 2}(y) d x d y \\
& =(2 t)^{n} \int_{\mathbb{R}^{n}}|\hat{g}(y)|^{2} d y,
\end{aligned}
$$

which leads to the identity, with $s=\frac{1}{2}$,

$$
\int_{\mathbb{C}^{n}}\left|u(z, t) e^{-\frac{i}{4 t} \sum z_{j}^{2}}\right|^{2} e^{-\frac{1}{4 t^{2}}|y|^{2}} d x d y=\left(4 t^{2} \pi\right)^{n / 2} \int_{\mathbb{R}^{n}}|f(y)|^{2} e^{|y|^{2}} d y .
$$

Actually, the converse is also true.

In this article we consider the Schrödinger equation associated to the Hermite and special Hermite operators and also to the sublaplacian on the Heisenberg group. 
The Hermite and Special Hermite cases are similar to the Euclidean Laplacian and straightforward, whereas the sublaplacian case is very different. We obtain an isometric isomorphism between certain subspaces of $L^{2}\left(H^{n}\right)$ and a direct integral of weighted Bergman spaces related to special Hermite operator.

The paper is organised as follows. In Sect. 2, we treat the Hermite and special Hermite cases. In Sect. 3, we consider the sublaplacian on the Heisenberg group. In Sect. 4, the generalized sublaplacian has been treated.

\section{Schrödinger equation for the Hermite and the special Hermite operators}

First we consider the regularising properties of the unitary group $e^{i t H}, t \in \mathbb{R}$ generated by the Hermite operator $H=-\Delta+|x|^{2}$ on $\mathbb{R}^{n}$. As is well known, $e^{i t H}$ is a fractional Fourier transform and hence the holomorphic extendability of $e^{i t H} f$ under suitable decay assumption on $f$ is only but expected. The spectral decomposition of $H$ is given by

$$
H=\sum_{k=0}^{\infty}(2 k+n) P_{k}
$$

where $P_{k} f=\sum_{|\alpha|=k}\left\langle f, \Phi_{\alpha}\right\rangle \Phi_{\alpha}, \Phi_{\alpha}$ being the normalised Hermite functions on $\mathbb{R}^{n}$. Using Mehler's formula (see [25]) we obtain

$$
e^{i t H} f(x)=\int_{\mathbb{R}^{n}} K_{i t}(x, y) f(y) d y
$$

where

$$
K_{i t}(x, y)=(2 \pi i \sin 2 t)^{-\frac{n}{2}} e^{\frac{i}{4}(\cot 2 t)\left(|x|^{2}+|y|^{2}\right)-i(\csc 2 t) x \cdot y} .
$$

Note that $K_{i t}(x, y)$ is well defined as long as $2 t$ is not an integral multiple of $\pi$.

The one parameter group $e^{i t H}$ is periodic in $t$ and for $2 t \neq k \pi, k \in \mathbb{Z}, e^{i t H}$ is given by the above integral. For such values of $t$,

$$
e^{-\frac{i}{4}(\cot 2 t)|x|^{2}} e^{i t H} f(x)=(2 \pi i \sin 2 t)^{-\frac{n}{2}} \int_{\mathbb{R}^{n}} f(y) e^{\frac{i}{4}(\cot 2 t)|y|^{2}} e^{-i(\csc 2 t) x \cdot y} d y .
$$

If we assume that $f(y) e^{s|y|^{2}} \in L^{2}\left(\mathbb{R}^{n}\right)$, then defining $g(y)=f(y) e^{s|y|^{2}} e^{\frac{i}{4}(\cot 2 t)|y|^{2}}$, we obtain

$$
e^{-\frac{i}{4}(\cot 2 t)|x|^{2}} e^{i t H} f(x)=(2 \pi i \sin 2 t)^{-\frac{n}{2}} \hat{g} * q_{s}((\csc 2 t) x)
$$

where $q_{s}$ is the Euclidean heat kernel on $\mathbb{R}^{n}$. Thus we see that $e^{i t H}$ is related to the Segal-Bargmann transform or the heat kernel transform associated to the standard Laplacian on $\mathbb{R}^{n}$. 
We briefly recall the main theorem on Segal-Bargmann transform for the convenience of the reader. The operator which takes $f \in L^{2}\left(\mathbb{R}^{n}\right)$ into the entire function $f * q_{t}(z)$ on $\mathbb{C}^{n}$ is called the Segal-Bargmann transform. This is a variant of the Bargmann transform studied by Bargmann [1] and independently by Segal. The main theorem proved in [1] can be restated as follows.

Theorem 2.1 The Segal-Bargmann transform takes $L^{2}\left(\mathbb{R}^{n}\right)$ onto the space of entire functions $F$ on $\mathbb{C}^{n}$ which are square integrable with respect to the measure $q_{t / 2}(y) d x d y$. Moreover, for any $f \in \mathfrak{七}^{2}\left(\mathbb{R}^{n}\right)$

$$
\int_{\mathbb{C}^{n}}\left|f * q_{t}(x+i y)\right|^{2} q_{t / 2}(y) d x d y=\int_{\mathbb{R}^{n}}|f(x)|^{2} d x .
$$

Using the above theorem of Segal and Bargmann we can easily prove the following result for the operators $e^{i t H}$.

Theorem 2.2 For any $t \neq \frac{k}{2} \pi, k \in \mathbb{Z}$, $e^{i t H}$ defines an isomorphism between $L^{2}\left(\mathbb{R}^{n}\right.$, $\left.e^{|x|^{2}} d x\right)$ and the Hilbert space of entire functions on $\mathbb{C}^{n}$ that are square integrable with respect to the weight function $(2 \pi|\sin 2 t|)^{-n} e^{-\left(\csc ^{2} 2 t\right)|y|^{2}} e^{(\cot 2 t) x \cdot y}$.

Proof As $g \in L^{2}\left(\mathbb{R}^{n}\right)$, the function $F(x, t)=e^{-\frac{i}{4}(\cot 2 t)|x|^{2}} e^{i t H} f(x)$ has an entire extension to $\mathbb{C}^{n}$ and according to the theorem of Bargmann, we have

$$
\begin{aligned}
& \int_{\mathbb{C}^{n}}|F(x+i y, t)|^{2} q_{s / 2}((\csc 2 t) y) d x d y \\
& =\left(2 \pi^{-n}\right)|\sin 2 t|^{n} \int_{\mathbb{C}^{n}}\left|\hat{g} * q_{s}(x+i y)\right|^{2} q_{s / 2}(y) d x d y \\
& =(2 \pi)^{-n}|\sin 2 t|^{n} \int_{\mathbb{R}^{n}}|\hat{g}(y)|^{2} d y \\
& =(2 \pi)^{-n}|\sin 2 t|^{n} \int_{\mathbb{R}^{n}}|f(y)|^{2} e^{2 s|y|^{2}} d y .
\end{aligned}
$$

Taking $s=\frac{1}{2}$ we observe that

$$
\begin{aligned}
& \int_{\mathbb{C}^{n}}|F(x+i y)|^{2} e^{-\left(\csc ^{2} 2 t\right)|y|^{2}} d x d y \\
& \quad=c_{n}(2 \pi)^{-n}|\sin 2 t|^{n} \int_{\mathbb{R}^{n}}|f(y)|^{2} e^{|y|^{2}} d y .
\end{aligned}
$$


This can be rewritten as

$$
\begin{aligned}
& \int_{\mathbb{C}^{n}}\left|e^{i t H} f(x+i y)\right|^{2} e^{(\cot 2 t) x \cdot y}|2 \pi \sin 2 t|^{-n} e^{-\left(\left(\csc ^{2} 2 t\right)|y|^{2}\right)} d x d y \\
& \quad=c_{n} \int_{\mathbb{R}^{n}}|f(y)|^{2} e^{|y|^{2}} d y .
\end{aligned}
$$

The converse follows from the fact that the Segal-Bargmann transform is onto. The proof of this fact is involved which uses the action of Bargmann transform on Hermite functions. See also Section 6, Chapter 1 of Folland [4] for details about Bargmann transform.

Remark 2.3 We remark that when $t=\frac{\pi}{4}, e^{i t H} f$ is a constant multiple of $\hat{f}$ and the above result reduces to

$$
\int_{\mathbb{R}^{n}} \int_{\mathbb{R}^{n}}|\hat{f}(x+i y)|^{2} e^{-|y|^{2}} d x d y=c_{n} \int_{\mathbb{R}^{n}}|f(y)|^{2} e^{|y|^{2}} d y .
$$

Therefore, we can view Theorem 2.1 as a result for the fractional Fourier transform.

We now turn our attention to the special Hermite operator $L_{\lambda}$ defined by

$$
L_{\lambda}=-\Delta+\frac{\lambda^{2}}{4}\left(|x|^{2}+|u|^{2}\right)-i \lambda \sum_{j=1}^{n}\left(x_{j} \partial_{u_{j}}-u_{j} \partial_{x_{j}}\right)
$$

where $\Delta$ is the Laplacian on $\mathbb{R}^{2 n}$ and $\lambda \in \mathbb{R} \backslash\{0\}$. The spectral decomposition of $L_{\lambda}$ is given by

$$
L_{\lambda} f=(2 \pi)^{-n} \sum_{k=0}^{\infty}(2 k+n)|\lambda| f *_{\lambda} \varphi_{k}^{\lambda}
$$

where the $\lambda$ twisted convolution between two functions is defined as

$$
f *_{\lambda} g(x, u)=\int_{\mathbb{C}^{n}} f\left(x-x^{\prime}, u-u^{\prime}\right) g\left(x^{\prime}, u^{\prime}\right) e^{i \frac{\lambda}{2}\left(u \cdot x^{\prime}-u^{\prime} \cdot x\right)} d x^{\prime} d u^{\prime},
$$

so that the Schrödinger equation is solved by the function

$$
e^{i t L_{\lambda}} f(x, u)=c_{\lambda} \sum_{k=0}^{\infty} e^{(2 k+n)|\lambda| i t} f *_{\lambda} \varphi_{k}^{\lambda}(x, u)
$$


Again the kernel of $e^{i t L_{\lambda}}$ is explicitly known in terms of the heat kernel $p_{t}^{\lambda}(x, u)$ associated to the special Hermite operator $L_{\lambda}$ (see [18]): $e^{i t L_{\lambda}} f=f *_{\lambda} p_{i t}^{\lambda}$, where

$$
p_{i t}^{\lambda}(x, u)=c_{n}\left(\frac{\lambda}{\sin \lambda t}\right)^{n} e^{-i \frac{\lambda}{4}(\cot t \lambda)\left(|x|^{2}+|u|^{2}\right)}
$$

As in the case of $H, e^{i t L_{\lambda}}$ is also periodic in $t$ and the above kernel exists as long as $t \lambda$ is not an integral multiple of $\pi$. Also note that for such $t, p_{i t}^{\lambda}(x, u)$ extends to $\mathbb{C}^{n} \times \mathbb{C}^{n}$ as an entire function.

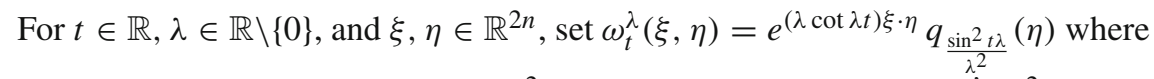
$q_{s}(\eta)$ is the Euclidean heat kernel on $\mathbb{R}^{2 n}$. Consider the Hilbert space $S_{t}^{\lambda}\left(\mathbb{C}^{2 n}\right)$ of all entire functions for which

$$
\|F\|_{S_{t}^{\lambda}}^{2}=\int_{\mathbb{C}^{2 n}}|F(\xi+i \eta)|^{2} \omega_{t}^{\lambda}(\xi, \eta) d \xi d \eta<\infty .
$$

We now prove

Theorem 2.4 The operator $e^{i t L_{\lambda}}$ is an isometric isomorphism between $L^{2}\left(\mathbb{R}^{2 n}\right.$, $\left.e^{|\xi|^{2}} d \xi\right)$ and the Hilbert space $S_{t}^{\lambda}\left(\mathbb{C}^{2 n}\right)$ :

$$
\int_{\mathbb{C}^{2 n}}\left|e^{i t L_{\lambda}} f(\xi+i \eta)\right|^{2} \omega_{t}^{\lambda}(\xi, \eta) d \xi d \eta=\int_{\mathbb{R}^{2 n}}|f(\xi)|^{2} e^{|\xi|^{2}} d \xi
$$

Moreover, the weight function $\omega_{t}^{\lambda}$ is unique.

Proof For $f \in L^{2}\left(\mathbb{R}^{2 n}, e^{|\xi|^{2}} d \xi\right)$, consider

$$
e^{i t L_{\lambda}} f(\xi)=f * \lambda p_{i t}^{\lambda}(\xi), \xi=(x, u)
$$

which is given by the integral

$$
c_{n}\left(\frac{\lambda}{\sin \lambda t}\right)^{n} \int_{\mathbb{R}^{2 n}} f(a, b) e^{i \frac{\lambda}{4}(\cot t \lambda)\left[|x-a|^{2}+|u-b|^{2}\right]} e^{i \frac{\lambda}{2}(a \cdot u-b \cdot x)} d a d b .
$$

Defining $v_{t}(\xi)=e^{i t L_{\lambda}} f(\xi) p_{-i t}^{\lambda}(\xi)$, we can write the above as

$$
v_{t}(\xi)=\left(\frac{\lambda}{\sin \lambda t}\right)^{2 n} \int_{\mathbb{R}^{2 n}} g_{\lambda}(\eta) e^{-i \frac{\lambda}{2}(A \eta \cdot \xi)} d \eta
$$

where $g_{\lambda}(\eta)=f(\eta) e^{i \frac{\lambda}{4}(\cot t \lambda)|\eta|^{2}}$ and $A$ is the $2 n \times 2 n$ matrix

$$
A=A(\lambda)=\left(\begin{array}{cc}
\cot (t \lambda) I_{n} & -I_{n} \\
I_{n} & \cot (t \lambda) I_{n}
\end{array}\right)
$$


We would like to express $v_{t}$ as the convolution of a function $h_{\lambda} \in L^{2}\left(\mathbb{R}^{2 n}\right)$ with the Euclidean heat kernel on $\mathbb{R}^{2 n}$, still denoted by $q_{s}$, for suitable $s$. Let $B=\left(A^{t}\right)^{-1}$ and $C=\frac{2}{\lambda} B$. We then have

$$
\begin{aligned}
v_{t}(\xi) & =\left(\frac{\lambda}{\sin \lambda t}\right)^{2 n} \int_{\mathbb{R}^{2 n}} g_{\lambda}(C \eta)|\operatorname{det} C| e^{-i \xi \cdot \eta} d \eta \\
& =\int_{\mathbb{R}^{2 n}} \hat{h_{\lambda}}(\eta) e^{-s|\eta|^{2}} e^{-i \xi \cdot \eta} d \eta
\end{aligned}
$$

where $\hat{h_{\lambda}}(\eta)=\left(\frac{\lambda}{\sin \lambda t}\right)^{2 n}|\operatorname{det} C| g_{\lambda}(C \eta) e^{s|\eta|^{2}}$ for a suitable $s$ to be chosen later.

Therefore, $v_{t}(\xi)=h_{\lambda} * q_{s}(\xi)$, the convolution with the Euclidean heat kernel on $\mathbb{R}^{2 n}$. If $h_{\lambda} \in L^{2}\left(\mathbb{R}^{2 n}\right)$, then it follows from the Bargmann-Segal theorem that $v_{t}(\xi)$ extends to $\mathbb{C}^{2 n}$ as an entire function and

$$
\int_{\mathbb{C}^{2 n}}\left|v_{t}(\xi+i \eta)\right|^{2} q_{s / 2}(\eta) d \xi d \eta=\int_{\mathbb{R}^{2 n}}\left|h_{\lambda}(\eta)\right|^{2} d \eta .
$$

Now,

$$
\begin{aligned}
\int_{\mathbb{R}^{2 n}}\left|h_{\lambda}(\eta)\right|^{2} d \eta & =\int_{\mathbb{R}^{2 n}}\left|\hat{h_{\lambda}}(\eta)\right|^{2} d \eta \\
& =\left(\frac{\lambda}{\sin \lambda t}\right)^{4 n}|\operatorname{det} C|^{2} \int_{\mathbb{R}^{2 n}}\left|g_{\lambda}(C \eta)\right|^{2} e^{2 s|\eta|^{2}} d \eta \\
& =\left(\frac{\lambda}{\sin \lambda t}\right)^{4 n}|\operatorname{det} C| \int_{\mathbb{R}^{2 n}}\left|g_{\lambda}(\eta)\right|^{2} e^{2 s\left|C^{-1} \eta\right|^{2}} d \eta
\end{aligned}
$$

After computing the determinant of $C$ and $\left|C^{-1} \eta\right|$ we end up with

$$
\begin{aligned}
\int_{\mathbb{R}^{2 n}}\left|h_{\lambda}(\eta)\right|^{2} d \eta & =\frac{\lambda^{4 n}}{(\sin t \lambda)^{4 n}} \frac{2^{2 n}}{\lambda^{2 n}}(\sin t \lambda)^{2 n} \int_{\mathbb{R}^{2 n}}\left|g_{\lambda}(\eta)\right|^{2} e^{\frac{\lambda^{2}}{2}|\eta|^{2} s \csc ^{2} t \lambda} d \eta \\
& =\left(\frac{2^{n} \lambda^{n}}{(\sin t \lambda)^{n}}\right)^{2 n} \int_{\mathbb{R}^{2 n}}|f(\eta)|^{2} e^{\left|\eta^{2}\right|} d \eta
\end{aligned}
$$

by choosing $s=2 \frac{\sin ^{2} t \lambda}{\lambda^{2}}$. Thus we have proved the isometry 


$$
\begin{aligned}
& \int_{\mathbb{C}^{2 n}}\left|e^{i t L_{\lambda}} f(\xi+i \eta)\right|^{2}\left|p_{-i t}^{\lambda}(\xi+i \eta)\right|^{2} q_{\frac{\sin ^{2}+\lambda}{\lambda^{2}}}(\eta) d \xi d \eta \\
& \quad=c_{\lambda} \int_{\mathbb{R}^{2 n}}|f(\eta)|^{2} e^{|\eta|^{2}} d \eta,
\end{aligned}
$$

where $c_{\lambda}=\left(\frac{2 \lambda}{\sin t \lambda}\right)^{2 n}$. Note that

$$
\left|p_{-i t}^{\lambda}(\xi+i \eta)\right|^{2}=\left(\frac{\lambda}{\sin t \lambda}\right)^{2 n}\left|e^{-i \frac{\lambda}{4}(\cot t \lambda)(\xi+i \eta)^{2}}\right|^{2}=\left(\frac{\lambda}{\sin t \lambda}\right)^{2 n} e^{\frac{\lambda}{2}(\cot t \lambda) \xi \cdot \eta}
$$

and hence $\omega_{t}^{\lambda}(\xi, \eta)=e^{\lambda(\cot t \lambda) \xi \cdot \eta} q_{\frac{\sin ^{2} t \lambda}{\lambda^{2}}}(\eta)$.

Observe that the equation

$$
v_{t}(\xi)=e^{i t L_{\lambda}} f(\xi) p_{-i t}^{\lambda}(\xi)=h_{\lambda} * q_{s}(\xi)
$$

with $s$ chosen as above sets up a one to one correspondence between the image of $L^{2}\left(\mathbb{R}^{2 n}, e^{|\xi|^{2}} d \xi\right)$ under $e^{i t L_{\lambda}}$ and the image of $L^{2}\left(\mathbb{R}^{2 n}\right)$ under the heat kernel transform viz. $h \rightarrow h * q_{s}(\xi+i \eta)$. Indeed, if

$$
F(\xi+i \eta)=h_{\lambda} * q_{s}(\xi+i \eta), h_{\lambda} \in L^{2}\left(\mathbb{R}^{2 n}\right)
$$

define $f$ in terms of $h_{\lambda}$ by the equations

$$
\hat{h_{\lambda}}(\eta)=|\operatorname{det} C| g_{\lambda}(C \eta) e^{s|\eta|^{2}}, \quad f(\eta)=g_{\lambda}(\eta) e^{-i \frac{\lambda}{4}(\cot t \lambda)|\eta|^{2}} .
$$

In other words,

$$
f(\eta)=\hat{h_{\lambda}}\left(C^{-1} \eta\right) e^{-s\left|C^{-1} \eta\right|^{2}}|\operatorname{det} C|^{-1} e^{-i \frac{\lambda}{4}(\cot t \lambda)|\eta|^{2}} .
$$

Since $2 s\left|C^{-1} \eta\right|^{2}=|\eta|^{2}$, we see that

$$
\int_{\mathbb{R}^{2 n}}|f(\eta)|^{2} e^{|\eta|^{2}} d \eta=c_{\lambda} \int_{\mathbb{R}^{2 n}}\left|\hat{h_{\lambda}}\left(C^{-1} \eta\right)\right|^{2} d \eta<\infty .
$$

Clearly,

$$
e^{i t L_{\lambda}} f(\xi) p_{-i t}^{\lambda}(\xi)=h_{\lambda} * q_{s}(\xi)=F(\xi)
$$

as the above calculation shows, and hence $e^{i t L_{\lambda}}$ is onto.

We now prove the uniqueness for the weight function. Let $\mathcal{F}_{s}\left(\mathbb{C}^{2 n}\right)$ be the image of $L^{2}\left(\mathbb{R}^{2 n}\right)$ under the transform $h \rightarrow h * q_{s}(\zeta)$ where $s=2 \frac{\sin ^{2} \lambda t}{\lambda^{2}}$. In view of the above remarks it is enough to prove the weight function characterising the space $\mathcal{F}_{s}\left(\mathbb{C}^{2 n}\right)$ 
is unique. We know from [1] that this space is a weighted Bergman space with the weight function $q_{a / 2}(\eta), \eta \in \mathbb{R}^{2 n}$. The space $\mathcal{F}_{s}$ is invariant under the action of $\mathbb{R}^{2 n}$. Consequently, if there exists another nonnegative weight function $W_{s}(\xi, \eta)$ such that

$$
\int_{\mathbb{C}^{2 n}}|G(\xi+i \eta)|^{2} W_{s}(\xi, \eta) d \xi d \eta=\int_{\mathbb{C}^{2 n}}|G(\xi+i \eta)|^{2} q_{s / 2}(\eta) d \xi d \eta
$$

then we should have $W_{s}(\xi, \eta)=W_{s}(\eta)([14$, p. 308]). Using the Plancherel formula in the $\xi$ variable we have

$$
\begin{aligned}
\int_{\mathbb{R}^{2 n}}|f(\xi)|^{2} d \xi & =\int_{\mathbb{C}^{2 n}}\left|f * q_{s}(\xi+i \eta)\right|^{2} W_{s}(\eta) d \xi d \eta \\
& =\int_{\mathbb{R}^{2 n}} \int_{\mathbb{R}^{2 n}}|\widehat{f}(\xi)|^{2} e^{-2 \xi \cdot \eta} e^{-2 s|\xi|^{2}} W_{s}(\eta) d \xi d \eta
\end{aligned}
$$

Taking $f$ to be the Gaussian $e^{-\frac{1}{2}|x|^{2}}$ we see that

$$
\int_{\mathbb{R}^{n}} W_{s}(\eta) e^{(1+2 s)^{-1}|\eta|^{2}} d \eta<\infty
$$

Moreover, $W_{s}(\eta)$ is given by the equation

$$
e^{2 s|\xi|^{2}}=\int_{\mathbb{R}^{2 n}} e^{-2 \xi \cdot \eta} W_{s}(\eta) d \eta
$$

In view of the above integrability of $W_{s}$, both sides of this equation extend to $\mathbb{C}^{n}$ as entire functions. Consequently we see that

$$
e^{-2 s|\xi|^{2}}=\int_{\mathbb{R}^{2 n}} e^{-2 i \xi \cdot \eta} W_{s}(\eta) d \eta
$$

and hence $W_{s}$ is equal to $q_{s / 2}(\eta)$. Thus the weight $\omega_{t}^{\lambda}$ is unique for $S_{t}^{\lambda}\left(\mathbb{C}^{2 n}\right)$.

\section{Schrödinger equation for the sublaplacian}

Let $X_{j}=\frac{\partial}{\partial x_{j}}-\frac{1}{2} u_{j} \frac{\partial}{\partial \xi}, U_{j}=\frac{\partial}{\partial u_{j}}+\frac{1}{2} x_{j} \frac{\partial}{\partial \xi}$ and $Z=\frac{\partial}{\partial \xi}$ be the left invariant vector fields on $H^{n}$ forming a basis for the Heisenberg Lie algebra. Then the sublaplacian on $H^{n}$ is defined by

$$
\mathcal{L}=-\sum_{j=1}^{n}\left(X_{j}^{2}+U_{j}^{2}\right)
$$


and it plays the role of Laplacian on $\mathbb{R}^{n}$. It is well known that $\mathcal{L}$ is a nonnegative essentially self adjoint operator which generates a semigroup $e^{-t \mathcal{L}}$ of convolution with the smooth kernel $p_{t}$ :

$$
p_{t}(x, u, \xi)=\int_{-\infty}^{\infty} e^{-i \lambda \xi} p_{t}^{\lambda}(x, u) d \lambda
$$

where $p_{t}^{\lambda}$ is the kernel associated to the special Hermite operator $L_{\lambda}$ studied in Sect. 2. Note that $e^{-t \mathcal{L}}$ and $e^{-t L_{\lambda}}$ are related via

$$
\left(e^{-t \mathcal{L}} f\right)^{\lambda}(x, u)=e^{-t L_{\lambda}} f^{\lambda}(x, u)
$$

Here $f^{\lambda}(x, u)=\int_{\mathbb{R}} f(x, u, \xi) e^{i \lambda \xi} d \xi$. At the convolution level $\left(f * p_{t}\right)^{\lambda}(x, u)=$ $f^{\lambda} * \lambda p_{t}^{\lambda}(x, u)$. In [14], the authors have characterised the image of $L^{2}\left(H^{n}\right)$ under the semigroup $e^{-t\left(\mathcal{L}+Z^{2}\right)}$. There it has been proved that the image is not a weighted Bergman space as expected, but can be expressed as a direct integral of weighted (twisted) Bergman spaces. Here we consider a similar problem for the Schrödinger equation and try to characterise the image of $L^{2}\left(H^{n}\right)$ under $e^{i t \mathcal{L}}$.

Formally $v_{t}(x, u, \xi)=e^{i t \mathcal{L}} f(x, u, \xi)$ solving the Schrödinger equation

$$
i \partial_{t} v_{t}(x, u, \xi)=\mathcal{L} v_{t}(x, u, \xi), v_{0}(x, u, \xi)=f(x, u, \xi)
$$

is given by $f * p_{i t}(x, u, \xi)$ which can also be written as

$$
f * p_{i t}(x, u, \xi)=\int_{-\infty}^{\infty} e^{-i \lambda \xi} f^{\lambda} * \lambda p_{i t}^{\lambda}(x, u) d \lambda .
$$

Note that the kernel $p_{i t}$ has singularities at the points where $\lambda t$ is an integral multiple of $\pi$. Therefore, the above formula is not valid even for fixed $t$, unless the function $\lambda \rightarrow f^{\lambda}$ is compactly supported so that the singularities are avoided. Observe that

$$
p_{t}^{\lambda}(x, u)=c_{n}\left(\frac{\lambda}{\sin t \lambda}\right)^{n} e^{-\frac{\lambda}{4}(\cot t \lambda)\left(|x|^{2}+|u|^{2}\right)}
$$

so that there is no problem at $\lambda=0$. Therefore, if we assume that $\lambda \rightarrow f^{\lambda}$ is supported in, say $|\lambda| \leq R$, the above integral representation for $f * p_{i t}$ makes sense for all $t,|t|<\frac{\pi}{R}$. If we further assume that $f^{\lambda}(x, u)$, has enough decay as a function of $(x, u)$, then $f * p_{i t}(x, u, \xi)$ can be holomorphically extended to the whole of $\mathbb{C}^{2 n+1}$ which is the complexification of $H^{n}$.

For $R>0$ let $\mathcal{H}_{R}$ stand for the Hilbert subspace of $L^{2}\left(H^{n}\right)$ consisting of functions $f$ having the following two properties:

(i) $f^{\lambda}$ is supported in $|\lambda| \leq R$

(ii) $\int_{\mathbb{R}^{2 n+1}}|f(x, u, \xi)|^{2} e^{\left(|x|^{2}+|u|^{2}\right)} d x d u d \xi<\infty$. 
Define $\|f\|_{\mathcal{H}_{R}}$ to be the square root of the quantity in (ii). Then $\mathcal{H}_{R}$ becomes a Hilbert space with the inner product

$$
(f, g)=\int_{\mathbb{R}^{2 n+1}} f(x, u, \xi) \overline{g(x, u, \xi)} e^{\left(|x|^{2}+|u|^{2}\right)} d x d u d \xi
$$

We have the following direct integral characterisation of the image of $\mathcal{H}_{R}$ under $e^{i t \mathcal{L}}$. Let $T_{t}$ stand for the map which takes $f \in L^{2}\left(H^{n}\right)$ into $\left(e^{i t L_{\lambda}} f^{\lambda}\right)_{\lambda \in[-R, R]}$.

Theorem 3.1 Let $R>0$ and $|t|<\frac{\pi}{R}$. Then $T_{t}: \mathcal{H}_{R} \rightarrow \int_{-R}^{R} \oplus S_{t}^{\lambda} d \lambda$ is, up to a constant multiple, an isometric isomorphism.

Proof The theorem follows from Theorem 2.4. Indeed, consider the map $f \rightarrow\left(e^{i t \mathcal{L}} f\right)^{\lambda}$ from $\mathcal{H}_{R} \rightarrow \int_{-R}^{R} \oplus S_{t}^{\lambda} d \lambda$. As $\left(e^{i t \mathcal{L}} f\right)^{\lambda}=e^{i t L_{\lambda}} f^{\lambda}$, we have

$$
\left\|e^{i t L_{\lambda}} f^{\lambda}\right\|_{S_{t}^{\lambda}}^{2}=c_{n} \int_{\mathbb{R}^{2 n}}\left|f^{\lambda}(x, u)\right|^{2} e^{\left(|x|^{2}+|u|^{2}\right)} d x d u
$$

Integrating with respect to $\lambda$ and using Plancherel in the $\xi$ variable, we obtain

$$
\int_{-R}^{R}\left\|\left(e^{i t \mathcal{L}} f\right)^{\lambda}\right\|_{S_{t}^{\lambda}}^{2} d \lambda=c_{n} \int_{\mathbb{R}^{2 n+1}}|f(x, u, \xi)|^{2} e^{\left(|x|^{2}+|u|^{2}\right)} d x d u d \xi .
$$

To prove the converse, suppose $\left(F_{\lambda}\right)_{\lambda \in[-R, R]}$ belong to $\int_{-R}^{R} \oplus S_{t}^{\lambda} d \lambda$. Then using Theorem 2.4 for each $\lambda \in[-R, R]$ there exists unique $f_{\lambda} \in L^{2}\left(\mathbb{R}^{2 n}, e^{|x|^{2}+\left|u^{2}\right|} d x d u\right)$ such that $e^{i t L_{\lambda}} f_{\lambda}=F_{\lambda}$ and the equality

$$
\int_{\mathbb{R}^{2 n}}\left|f_{\lambda}(x, u)\right|^{2} e^{|x|^{2}+\left|u^{2}\right|} d x d u=\left\|F_{\lambda}\right\|_{S_{t}^{\lambda}}^{2}
$$

holds. We define $f$ on $H^{n}$ by

$$
f(x, u, t)=\int_{-R}^{R} f_{\lambda}(x, u) e^{-i \lambda t} d \lambda
$$

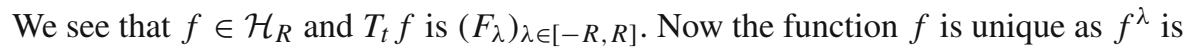
so. This proves the surjectivity.

We now address the problem of characterising the image of $\mathcal{H}_{R}$ under $e^{i t \mathcal{L}}$ as a weighted Bergman space. Note that the operator $e^{i t \mathcal{L}}$ commutes with left translations by elements of the Heisenberg group (being a right convolution operator) but the space 
$\mathcal{H}_{R}$ is not invariant under left translations. However, it is invariant under translation in the last variable. This means that $\mathcal{H}_{R}$ is invariant under translation by elements of the centre of the Heisenberg group. This shows that the image of $\mathcal{H}_{R}$ under $e^{i t \mathcal{L}}$ is also invariant under the action of centre of Heisenberg group. Suppose now that there is a nonnegative weight function $W_{t}(z, w, s)$ on $\mathbb{C}^{n} \times \mathbb{C}^{n} \times \mathbb{C}$ such that

$$
\begin{aligned}
& \int_{\mathbb{C}^{2 n+1}}\left|e^{i t \mathcal{L}} f(z, w, \zeta)\right|^{2} W_{t}(z, w, \zeta) d z d w d \zeta \\
& =c_{n} \int_{\mathbb{R}^{2 n+1}}|f(x, u, \xi)|^{2} e^{\left(|x|^{2}+|u|^{2}\right)} d x d u d \xi
\end{aligned}
$$

for all $f \in \mathcal{H}_{R}$. Replacing $f$ by $\tau_{a} f(x, u, \xi)=f(x, u, \xi-a), a \in \mathbb{R}$ and using the fact that $e^{i t \mathcal{L}}$ commutes with $\tau_{a}$ we see that

$$
\begin{gathered}
\int_{\mathbb{C}^{2 n+1}}\left|e^{i t \mathcal{L}} f(z, w, \zeta)\right|^{2} W_{t}(z, w, \zeta+a) d z d w d \zeta \\
=c_{n} \int_{\mathbb{R}^{2 n+1}}|f(x, u, \xi)|^{2} e^{\left(|x|^{2}+|u|^{2}\right)} d x d u d \xi
\end{gathered}
$$

for all $f \in \mathcal{H}_{R}$ and $a \in R$. Since the right hand side is independent of $a$ we see that $W_{t}(z, w, \zeta+a)=W_{t}(z, w, \zeta)$ for all $a$ which means that $W_{t}(z, w, \zeta)$ depends only on the imaginary part of $\zeta$. Thus if we let $\zeta=\xi+i \eta$ then

$$
\begin{gathered}
\int_{\mathbb{C}^{2 n+1}}\left|e^{i t \mathcal{L}} f(z, w, \zeta)\right|^{2} W_{t}(z, w, i \eta) d z d w d \zeta \\
=c_{n} \int_{\mathbb{R}^{2 n+1}}|f(x, u, \xi)|^{2} e^{\left(|x|^{2}+|u|^{2}\right)} d x d u d \xi
\end{gathered}
$$

Using the Euclidean Plancherel theorem in the $\xi$ variable, the above leads to

$$
\begin{aligned}
& \int_{\mathbb{R}} \int_{\mathbb{R}} \int_{\mathbb{C}^{2 n}}\left|e^{i t L_{\lambda}} f^{\lambda}(z, w)\right|^{2} e^{2 \lambda \eta} W_{t}(z, w, i \eta) d z d w d \eta d \lambda \\
& =c_{n} \int_{\mathbb{R}} \int_{\mathbb{R}^{2 n}}\left|f^{\lambda}(x, u)\right|^{2} e^{\left(|x|^{2}+|u|^{2}\right)} d x d u d \lambda .
\end{aligned}
$$

Let $\varphi_{k}$ be a sequence of Schwartz functions on $\mathbb{R}$ such that $\left(\hat{\varphi}_{k}\right)^{2}$ converges to the Dirac mass at $\lambda_{0}$. Replacing $f$ by $f(x, u, \xi) \varphi_{k}(\xi)$ in the above equation and letting $k$ 
tend to infinity we obtain

$$
\begin{aligned}
& \int_{\mathbb{R}} \int_{\mathbb{C}^{2 n}}\left|e^{i t L_{\lambda_{0}}} f^{\lambda_{0}}(z, w)\right|^{2} e^{2 \lambda_{0} \eta} W_{t}(z, w, i \eta) d z d w d \eta \\
& \quad=c_{n} \int_{\mathbb{R}^{2 n}}\left|f^{\lambda_{0}}(x, u)\right|^{2} e^{\left(|x|^{2}+|u|^{2}\right)} d x d u
\end{aligned}
$$

This is true for any $\lambda_{0},\left|\lambda_{0}\right| \leq R$. Since the weight function for the image of $L^{2}\left(\mathbb{R}^{2 n}\right)$ under $e^{i t L_{\lambda}}$ is unique, the above leads to the condition

$$
\begin{aligned}
& \int_{-\infty}^{\infty} e^{2 \lambda \eta} W_{t}(z, w, i \eta) d \eta \\
& =c_{n}\left(\frac{\lambda^{2}}{\sin ^{2} t \lambda}\right)^{n} e^{-\frac{1}{4}\left(\frac{\lambda^{2}}{\sin ^{2} t \lambda}\right)\left(|y|^{2}+|v|^{2}\right)} e^{\lambda(\cot t \lambda)(x \cdot y+u \cdot v)}
\end{aligned}
$$

which should be valid for all $|\lambda| \leq R$.

We now show that the above condition cannot be satisfied by a non negative weight function leading to the following negative result.

Theorem 3.2 There does not exist a non negative weight function $W_{t}(z, w, i \eta)$ such that

$$
\begin{gathered}
\int_{\mathbb{C}^{2 n+1}}\left|e^{i t \mathcal{L}} f(z, w, \zeta)\right|^{2} W_{t}(z, w, i \eta) d z d w d \zeta \\
=c_{n} \int_{\mathbb{R}^{2 n+1}}\left|f^{\lambda}(x, u)\right|^{2} e^{\left(|x|^{2}+|u|^{2}\right)} d x d u d \lambda
\end{gathered}
$$

for all $f \in \mathcal{H}_{R}$.

Proof As we have observed, the weight function has to satisfy the condition (3.5) for all $|\lambda| \leq R$. We fix $z$ and $w$ and consider $W_{t}(z, w, i \eta)$ as a function of $\eta$. In view of (3.5) it is integrable as a function of $\eta$ as we are assuming that $W_{t}$ is nonnegative.

Moreover,

$$
(\lambda+i s) \rightarrow \int_{\mathbb{R}} e^{2(\lambda+i s) \eta} W_{t}(z, w, i \eta) d \eta
$$

is holomorphic on the strip $|\lambda|<R$. To see this, we need only to verify the convergence of the integral

$$
\int_{\mathbb{R}}|\eta| e^{2 \lambda \eta} W_{t}(z, w, i \eta) d \eta
$$


But when $|\lambda|<R$ we can choose $\epsilon>0$ so that $|\lambda \pm \epsilon|<R$ and since $|\eta| \leq C_{\epsilon} e^{2 \epsilon|\eta|}$ we get

$$
\int_{\mathbb{R}}|\eta| e^{2 \lambda \eta} W_{t}(z, w, i \eta) d \eta \leq C_{\epsilon} \int_{\mathbb{R}} e^{2(\lambda \pm \epsilon) \eta} W_{t}(z, w, i \eta) d \eta<\infty .
$$

The right hand side of (3.5) is also holomorphic on $|\lambda|<R$ since $|t \lambda|<\pi$. Therefore, we have

$$
\begin{aligned}
& \int e^{2 i s \eta} W_{t}(z, w, i \eta) d \eta \\
& =c_{n}\left(\frac{s^{2}}{\sinh ^{2} t s}\right)^{n} e^{-\frac{1}{4}\left(\frac{s^{2}}{\sinh ^{2} t s}\right)\left(|y|^{2}+|v|^{2}\right)} e^{-s(\operatorname{coth} t s)(x \cdot y+u \cdot v)}
\end{aligned}
$$

for all $s \in \mathbb{R}$.

The above condition cannot be satisfied by an integrable $W_{t}(z, w, i \eta)$ in view of the fact that Fourier transform of an integrable function is bounded. Indeed, as $s \rightarrow \infty$, $\operatorname{coth} t s \rightarrow 1$ and $\frac{s^{2}}{\sinh ^{2} t s} \rightarrow 0$ at the fixed exponential rate $e^{-2 t s}$. Therefore, when $x \cdot y+u \cdot v$ is large and negative the right hand side of (3.6) becomes unbounded. This proves the nonexistence of $W_{t}$.

We conclude this section with the following remarks. Instead of looking for a weight function satisfying (3.5) we might consider the condition

$$
\begin{aligned}
& \int_{-\infty}^{\infty} e^{2 \lambda \eta} W_{t}(z, w, i \eta) d \eta \\
& =c_{n}\left(\frac{\lambda^{2}}{\sin ^{2} t \lambda}\right)^{n} e^{2 \epsilon \lambda^{2}} e^{-\frac{1}{4}\left(\frac{\lambda^{2}}{\sin ^{2} t \lambda}\right)\left(|y|^{2}+|v|^{2}\right)} e^{\lambda(\cot t \lambda)(x \cdot y+u \cdot v)}
\end{aligned}
$$

where $\epsilon>0$. Again the above is required to be valid only for $|\lambda| \leq R$. A weight function $W_{t}^{\epsilon}(z, w, i \eta)$ satisfying the above condition can be obtained by inverting a Laplace transform. It can be shown that the function

$$
\begin{aligned}
& W_{t}^{\epsilon}(z, w, \xi+i \eta) \\
& =\int_{\mathbb{R}} e^{2 \epsilon\left(\lambda+\frac{i}{2} s\right)^{2}} e^{-2 \eta\left(\lambda+\frac{i}{2} s\right)} \omega_{t}^{\lambda+\frac{i}{2} s}(z, w) d s
\end{aligned}
$$

is well defined, independent of the parameter $\lambda$ and satisfies the above condition. However, we do not know if the resulting function is non-negative or not. Nevertheless, we can proceed as in [14] where a similar situation was encountered. It is possible to use $W_{t}^{\epsilon}$ to define a positive definite sesquilinear form and then to prove that 


$$
\begin{gathered}
\int_{\mathbb{C}^{2 n+1}}\left|e^{-i t \mathcal{L}} f(z, w, \zeta)\right|^{2} W_{t}^{\epsilon}(z, w, i \eta) d z d w d \zeta \\
=c_{n} \int_{\mathbb{R}^{2 n+1}}\left|f^{\lambda}(x, u)\right|^{2} e^{\epsilon \lambda^{2}} e^{\left(|x|^{2}+|u|^{2}\right)} d x d u d \lambda .
\end{gathered}
$$

\section{Schrödinger equation for the generalized sublaplacians}

In Sect. 3 we defined the sublaplacian $\mathcal{L}$ for the Heisenberg group $H^{n}$ as

$$
\mathcal{L}=-\sum_{j=1}^{n}\left(X_{j}^{2}+U_{j}^{2}\right)
$$

which has the following explicit expression:

$$
\mathcal{L}=-\Delta-\frac{1}{4}\left(|x|^{2}+|u|^{2}\right) \frac{\partial^{2}}{\partial \xi^{2}}+\sum_{j=1}^{n}\left(x_{j} \frac{\partial}{\partial u_{j}}-u_{j} \frac{\partial}{\partial x_{j}}\right) \frac{\partial}{\partial \xi} .
$$

The radial part of $\mathcal{L}$ denoted by $\mathcal{L}_{n-1}$ can be easily calculated to be

$$
\mathcal{L}_{n-1}=-\left(\frac{\partial^{2}}{\partial r^{2}}+\frac{(2 n-1)}{r} \frac{\partial}{\partial r}+\frac{1}{4} r^{2} \frac{\partial^{2}}{\partial \xi^{2}}\right) .
$$

In this section we are interested in investigating analyticity of solutions of the Schrödinger equation associated with the generalized sublaplacian

$$
\mathcal{L}_{\alpha}=-\left(\frac{\partial^{2}}{\partial r^{2}}+\frac{2 \alpha+1}{r} \frac{\partial}{\partial r}+\frac{1}{4} r^{2} \frac{\partial^{2}}{\partial \xi^{2}}\right) .
$$

The operators $\mathcal{L}_{\alpha}$ have been studied extensively by many authors [21,24]. For each $\lambda \neq 0$ and $\alpha \geq 0$ we define the operators $\mathcal{L}_{\alpha}^{\lambda}$ by the relation:

$$
\left(\mathcal{L}_{\alpha} f\right)^{\lambda}(r)=\mathcal{L}_{\alpha}^{\lambda} f^{\lambda}(r)
$$

where $f^{\lambda}(r)=\int_{\mathbb{R}} e^{i \lambda t} f(r, t) d t$. It is to be noted that $\mathcal{L}_{\alpha}^{\lambda}$ is nothing but $\lambda$ scaled Laguerre operator

$$
\mathcal{L}_{\alpha}^{1}=-\frac{\partial^{2}}{\partial r^{2}}-\frac{(2 \alpha+1)}{r} \frac{\partial}{\partial r}+\frac{1}{4} r^{2}
$$

of type $\alpha$. Thus in order to study the operators $\mathcal{L}_{\alpha}^{\lambda}$ for fixed $\lambda \neq 0$ it is sufficient to consider $\mathcal{L}_{\alpha}^{1}$ only. Consider the Laguerre functions $\psi_{k}^{\alpha}(r)=\left(\frac{2^{-\alpha} \Gamma(k+1)}{\Gamma(k+\alpha+1)}\right)^{1 / 2}$ $L^{\alpha}\left(\frac{1}{2} r^{2}\right) e^{-\frac{1}{4} r^{2}}, k \geq 0$. Then $\psi_{k}^{\alpha}(r)$ is an eigenfunction of $\mathcal{L}_{\alpha}^{1}$ with eigenvalue $(2 k+\alpha+1)$. 
Moreover, these functions form an orthonormal basis for $L^{2}\left(\mathbb{R}_{+}, r^{2 \alpha+1}\right)$. We refer to [25] for more about Laguerre functions. Consequently, $\psi_{k, \lambda}^{\alpha}(r)=|\lambda|^{\frac{(\alpha+1)}{2}} \psi_{k}^{\alpha}(\sqrt{\mid} \lambda \mid r)$ are eigenfunctions of $\mathcal{L}_{\alpha}^{\lambda}$ with eigenvalues $(2 k+\alpha+1)|\lambda|$.

The images of $L^{2}$ under the semigroups for Bessel, Hermite, Laguerre operators have been characterized in $[2,7,12,27]$. We will be using some results stated and proved there. The Laguerre semigroup $e^{-t \mathcal{L}_{\alpha}^{1}}, t>0$ is an integral operator and can be expressed by

$$
e^{-t \mathcal{L}_{\alpha}^{1}} f(x)=\int_{0}^{\infty} K_{t}(x, \xi) f(\xi) \xi^{2 \alpha+1} d \xi
$$

where $K_{t}(x, \xi)=c_{\alpha}(\sinh (t))^{-1} e^{-\frac{1}{4} \operatorname{coth} t\left(x^{2}+\xi^{2}\right)} \frac{J_{\alpha}\left(\frac{i x \xi}{2 \sinh (t)}\right)}{(x \xi)^{\alpha}}$. Define $g(x)=$ $f(x) e^{-\tanh (t / 2) x^{2}}$. Then the relation between Laguerre and Bessel semigroup will give us

$$
e^{\frac{1}{2} \tanh \left(\frac{t}{2}\right) x^{2}} e^{-t \mathcal{L}_{\alpha}^{1}} f(x)=e^{-\sinh (t) B_{\alpha}^{1}} g(x)
$$

Here the Bessel semigroup $e^{-t B_{\alpha}^{1}} g$ of type $\alpha$ is given by the following integral operator

$$
e^{-t B_{\alpha}^{1}} g(x)=\int_{0}^{\infty} b_{t}(x, \xi) g(\xi) \xi^{2 \alpha+1} d \xi
$$

where the kernel $b_{t}$ is given by

$$
b_{t}(x, \xi)=c_{\alpha}(2 t)^{-1}(x \xi)^{-\alpha} J_{\alpha}\left(\frac{i x \xi}{2 t}\right) e^{-\frac{1}{4 t}\left(x^{2}+\xi^{2}\right)} .
$$

In order to study the semigroup associated with Laguerre operator it is necessary to study the same associated to the Bessel operator. We introduce the Bessel function of the third kind known as McDonald function $K_{\alpha}$ defined by

$$
K_{\alpha}(z)=\left(\frac{\pi}{2 z}\right)^{1 / 2} \frac{e^{-z}}{\Gamma\left(\alpha+\frac{1}{2}\right)} \int_{0}^{\infty} e^{-t} t^{\alpha-\frac{1}{2}}\left(1+\frac{t}{2 z}\right)^{\alpha-\frac{1}{2}} d t .
$$

We refer to [3] for properties of McDonald function $K_{\alpha}$. We define $k_{t, \alpha}(u, v)=$ $\frac{2^{\alpha}}{\pi}\left(\frac{u^{2}+v^{2}}{2 t}\right)^{2 \alpha+1} K_{\alpha+\frac{1}{2}}\left(\frac{1}{2 t}\left(u^{2}+v^{2}\right)\right)$ and $h_{t, \alpha}(u, v)=e^{\frac{1}{4 t}\left(u^{2}-v^{2}\right)} k_{t, \alpha}(u, v)$. The following theorem characterize the image of $L^{2}\left(\mathbb{R}^{+}, r^{2 \alpha+1}\right)$ under the Bessel semigroup (see [7, p. 184]). 
Theorem 4.1 For $f \in L^{2}\left(\mathbb{R}^{+}, r^{2 \alpha+1} d r\right)$ the function $e^{-t B_{\alpha}^{1}} f(u)$ extends to $\mathbb{C}$ as an even entire function and

$$
\int_{\mathbb{C}}\left|e^{-t B_{\alpha}^{1}}(f)(u+i v)\right|^{2} h_{t, \alpha}(u, v) d u d v=\int_{0}^{\infty}|f(r)|^{2} r^{2 \alpha+1} d r .
$$

Moreover, the map $f(r) \mapsto e^{-t B_{\alpha}^{1}} f(u+i v)$ takes $L^{2}\left(\mathbb{R}^{+}, r^{2 \alpha+1} d r\right)$ onto the space of even entire functions $F$ for which $\int_{\mathbb{C}}|F(u+i v)|^{2} h_{t, \alpha}(u, v) d u d v<\infty$.

Take $t=1$. Then any radial weight function $h(|w|)$ having the property

$$
\int_{0}^{\infty}|f(r)|^{2} r^{2 \alpha+1} d r=\int_{\mathbb{C}}\left|e^{-B} f(w)\right|^{2}\left|e^{\frac{1}{4} w^{2}}\right| h(|w|) d w
$$

for all $f$ satisfies the equations

$$
\int_{\mathbb{C}}|w|^{4 k} h(|w|) d w=2^{1+2 \delta} 2^{4 k} \Gamma(k+1) \Gamma(k+\alpha+1)
$$

for all $k \in \mathbb{N} \cup\{0\}$, (see [7, p. 184]). We will show that such a weight function is unique. We can write the above identity as

$$
(2 \pi) \int_{0}^{\infty} r^{4 k+1} h(r) d r=2^{1+2 \delta} 2^{4 k} \Gamma(k+1) \Gamma(k+\alpha+1) .
$$

Thus

$$
\int_{0}^{\infty} r^{k} d \mu(r)=2^{4 k} \Gamma(k+1) \Gamma(k+\alpha+1)=c(k)
$$

where $\mu$ is the measure given by

$$
\mu[0, r)=2^{-(1+2 \delta)} \frac{2}{\pi} \int_{0}^{r} \frac{h\left(s^{\frac{1}{4}}\right)}{s^{\frac{1}{2}}} d s .
$$

Note that $c_{k} \leq 2^{4 k}\{\Gamma(k+\alpha+1)\}^{2}$. We will use the following Theorem, known as Carleman's criteria, for Stieltje's moment problem ([20, Theorem 1.11]) to prove the uniqueness of $h$.

Theorem 4.2 Let $\Psi$ be a nondecreasing function defined on the interval $[0, \infty)$ such that its moments $\int_{0}^{\infty} r^{k} d \Psi(r), k=0,1,2, \cdots$, have a prescribed set of values 
$\beta_{k}, k=0,1,2, \cdots$ A sufficient condition that the function $\Psi$ is uniquely determined is that $\sum_{k=1}^{\infty} \beta_{k}^{-\frac{1}{2 k}}=\infty$.

In order to achieve the uniqueness of $h$ we need to show that $\sum_{k=0}^{\infty} c_{k}^{-\frac{1}{2 k}}=\infty$. Let $d_{k}=2^{4 k}\{\Gamma(k+\alpha+1)\}^{2}$. By Stirling's formula there exist $A, B>0$ such that $A d_{k} \leq 2^{4 k}\left\{e^{-(k+\alpha)}(k+\alpha)^{k+\alpha+\frac{1}{2}}\right\}^{2} \leq B d_{k}$. Since $c_{k} \leq d_{k}$ it follows that

$$
\begin{aligned}
c_{k}^{-\frac{1}{2 k}} & \geq d_{k}^{-\frac{1}{2 k}} \\
& \geq A^{\frac{1}{2 k}} 2^{-2} e(k+\alpha)^{-1-\frac{2 \alpha+1}{2 k}} .
\end{aligned}
$$

Let $a_{k}=(k+\alpha)^{-1-\frac{2 \alpha+1}{2 k}}$. The series $\sum_{k=0}^{\infty} c_{k}^{-\frac{1}{2 k}}$ will be divergent if we can show that the series $\sum_{k=0}^{\infty} a_{k}$ is divergent. Now $a_{k}>a_{k+1}$ and we have the inequalities

$$
2^{\frac{k}{2^{k}}}<\left(2^{k}+\alpha\right)^{\frac{1}{2^{k}}}<2^{\frac{k}{2^{k}}}(1+\alpha)^{\frac{1}{2^{k}}}
$$

Hence we have $\lim _{k \rightarrow \infty} 2^{k} a_{2^{k}}=\lim _{k \rightarrow \infty} \frac{2^{k}}{2^{k}+\alpha}\left(\left(2^{k}+\alpha\right)^{\frac{1}{2^{k}}}\right)^{-(2 \alpha+1)}=1$ which leads to the conclusion that the series $\sum_{k=1}^{\infty} 2^{k} a_{2^{k}}$ is divergent and hence by Theorem 3.27 of [19] the series $\sum_{k=0}^{\infty} a_{k}$ is also divergent. Thus we conclude that $\sum_{k=0}^{\infty} c_{k}^{-\frac{1}{2 k}}$ is a divergent series. This proves the uniqueness.

We first study the Schrödinger equation for the Bessel operators $B_{\alpha}^{1}$ and this will be used to study the same for Laguerre operators $\mathcal{L}_{\alpha}^{1}$. If $V_{t}^{1} f$ denotes the solution of the Schrödinger equation

$$
i \partial_{t} u=B_{\alpha}^{1} u, u(x, 0)=f(x)
$$

then it is not hard to see that

$$
e^{-i \frac{1}{4 t} x^{2}} V_{t}^{1} f(x)=c(\alpha)(2 t)^{-1} \int_{0}^{\infty} e^{i \frac{1}{4 t} \xi^{2}} f(\xi) \frac{J_{\alpha}\left(\frac{x \xi}{2 t}\right)}{(x \xi)^{\alpha}} \xi^{2 \alpha+1} d \xi
$$

Like in the Euclidean case $e^{-i \frac{1}{4 t} x^{2}} V_{t}^{1} f(x)$ is the Hankel transform (of type $\alpha$ ) of $e^{\frac{i}{4 t} \xi^{2}} f(\xi)$. Hence, if we assume that $f$ has Gaussian decay we can expect a holomorphic extension of $V_{t}^{1} f$ and an isometry as stated in the following theorem:

Theorem 4.3 $V_{t}^{1} f$ can be extended holomorphically to $\mathbb{C}$ provided $f$ satisfies $\int_{0}^{\infty}$ $|f(\xi)|^{2} e^{2 s \xi^{2}} \xi^{2 \alpha+1}<\infty$, for some $s>0$. Also we have the following isometry property:

$$
(2 t)^{2 \alpha} \int_{\mathbb{C}}\left|V_{t}^{1}(f)(x+i y)\right|^{2} w_{s, t, \alpha}(x, y) d z=c_{\alpha} \int_{0}^{\infty}|f(\xi)|^{2} e^{2 s \xi^{2}} \xi^{2 \alpha+1} d \xi,
$$


where $w_{s, t, \alpha}(x, y)=e^{\frac{1}{t} x y} h_{s, \alpha}\left(\frac{1}{2 t}(x, y)\right)$. Moreover, the map $f \mapsto V_{t}^{1}(f)$ takes $L^{2}\left(\mathbb{R}^{+}, e^{2 s \xi^{2}} \xi^{2 \alpha+1} d r\right)$ onto the space of even entire functions $F$ for which

$$
\int_{\mathbb{C}}|F(x+i y)|^{2} w_{s, t, \alpha}(x, y)<\infty .
$$

Proof From our assumption the function $e^{i \frac{1}{4 t} \xi^{2}} f(\xi) e^{s \xi^{2}}$ is in $L^{2}\left(\mathbb{R}_{+}, \xi^{2 \alpha+1}\right)$. Let $p_{s, \alpha}$ be the heat kernel for the Bessel operator so that $\mathcal{H}^{\alpha}\left(p_{s, \alpha}\right)(\xi)=e^{-s \xi^{2}}$. From the Plancherel theorem for the Hankel transform $\mathcal{H}^{\alpha}$ we get a function $g \in L^{2}\left(\mathbb{R}_{+}, \xi^{2 \alpha+1}\right)$ such that $\mathcal{H}^{\alpha} g(\xi)=e^{i \frac{1}{4 t} \xi^{2}} f(\xi) e^{s \xi^{2}}$. Then

$$
\begin{aligned}
& e^{-i \frac{1}{4 t}(2 t x)^{2}} V_{t}^{1} f(2 t x) \\
& =c_{\alpha}(2 t)^{-(\alpha+1)} \int_{0}^{\infty} \mathcal{H}^{\alpha} g(\xi) \mathcal{H}^{\alpha} p_{s, \alpha}(\xi) \frac{J_{\alpha}(x \xi)}{(x \xi)^{\alpha}} \xi^{2 \alpha+1} d \xi \\
& =c_{\alpha}(2 t)^{-(\alpha+1)} e^{-s B_{\alpha}^{1} g(x) .}
\end{aligned}
$$

Thus the problem reduces to the problem of characterizing the image of $L^{2}$ under the heat semigroup for the Bessel operator. Therefore,

$$
\begin{aligned}
& (2 t)^{2(\alpha+1)} \int_{\mathbb{C}}\left|e^{-\frac{i}{4 t}(2 t(x+i y))^{2}} V_{t}^{1} f(2 t(x+i y))\right|^{2} h_{s, \alpha}(x, y) d x d y \\
& =c_{\alpha} \int_{\mathbb{C}}\left|e^{-s B_{\alpha}^{1}} g(x+i y)\right|^{2} h_{s, \alpha}(x, y) d x d y \\
& =c_{\alpha} \int_{0}^{\infty}|g(\xi)|^{2} \xi^{2 \alpha+1} \\
& =c_{\alpha} \int_{0}^{\infty}|f(\xi)|^{2} e^{2 s \xi^{2}} \xi^{2 \alpha+1} d \xi
\end{aligned}
$$

We get the required result by a change of variable of the integral in the left hand side.

Corollary 4.4 By taking $s=\frac{1}{4}$ in the above theorem we get the following

$$
(2 t)^{2 \alpha} \int_{\mathbb{C}}\left|V_{t}^{1} f(x+i y)\right|^{2} w_{t, \alpha}(x, y) d x d y=c_{\alpha} \int_{0}^{\infty}|f(\xi)|^{2} e^{\frac{\xi^{2}}{2}} \xi^{2 \alpha+1} d \xi
$$

where $w_{t, \alpha}(x, y)=e^{\frac{1}{t} x y} h_{t^{2}, \alpha}(x, y)$. 
Let $f$ and $g$ be two functions related by $g(x)=f(x) e^{-i \tan \left(\frac{t}{2}\right) x^{2}}$. Then we can see that

$$
e^{i \frac{1}{2} \tan \left(\frac{t}{2}\right) x^{2}} e^{-i t \mathcal{L}_{\alpha}^{1}} f(x)=V_{\sin t}^{1} g(x)
$$

Using Corollary 4.4 above we get

$$
\begin{aligned}
& c_{\alpha} \int_{0}^{\infty}|f(\xi)|^{2} e^{\frac{\xi^{2}}{2}} \xi^{2 \alpha+1} d \xi \\
& =c_{\alpha} \int_{0}^{\infty}|g(\xi)|^{2} e^{\frac{\xi^{2}}{2}} \xi^{2 \alpha+1} d \xi \\
& =(2 \sin t)^{2 \alpha} \int_{\mathbb{C}}\left|V_{\sin t}^{1} g(x+i y)\right|^{2} w_{\sin t, \alpha}(x, y) d x d y \\
& =(2 \sin t)^{2 \alpha} \int_{\mathbb{C}}\left|e^{i \frac{1}{2} \tan \left(\frac{t}{2}\right)(x+i y)^{2}} e^{-i t \mathcal{L}_{\alpha}^{1}} f(x+i y)\right|^{2} w_{\sin t, \alpha}(x, y) d x d y \\
& =(2 \sin t)^{2 \alpha} \int_{\mathbb{C}}\left|e^{-i t \mathcal{L}_{\alpha}^{1}} f(x+i y)\right|^{2} e^{-2 \tan \left(\frac{t}{2}\right) x y} e^{\frac{1}{\sin t} x y} h_{\sin ^{2} t, \alpha}(x, y) d x d y \\
& =(2 \sin t)^{2 \alpha} \int_{\mathbb{C}}\left|e^{-i t \mathcal{L}_{\alpha}^{1}} f(x+i y)\right|^{2} e^{(2 \cot t-\csc t) x y} h_{\sin ^{2} t, \alpha}(x, y) d x d y .
\end{aligned}
$$

We define $\theta_{t, \alpha}(x, y)=(2 \sin t)^{2 \alpha} e^{(2 \cot t-\csc t) x y} h_{\sin ^{2} t, \alpha}(x, y)$. Let $\mathcal{A}_{t}^{\alpha}(\mathbb{C})$ stand for the space of all even entire functions $F$ on $\mathbb{C}$ for which

$$
\int_{\mathbb{C}}|F(x+i y)|^{2}\left\{\Theta_{t, \alpha}(x+i y) d x d y<\infty\right\} .
$$

With this definition of the weight we have the following theorem for the operator $\mathcal{L}_{\alpha}^{1}$.

Theorem 4.5 For any $t \in \mathbb{R}$ with $t \neq m \pi$, $e^{-i t \mathcal{L}_{\alpha}^{1}}$ defines an onto isomorphism between $L^{2}\left(\mathbb{R}_{+}, e^{\frac{\xi^{2}}{2}} \xi^{2 \alpha+1}\right)$ and the Hilbert space $\mathcal{A}_{t}^{\alpha}(\mathbb{C})$ and moreover the weight function is unique.

In order to study the Schrödinger equation associated to $\mathcal{L}_{\alpha}^{\lambda}$ we use the following relation:

$$
e^{-i t \mathcal{L}_{\alpha}^{\lambda}} f(r)=e^{-i|\lambda| t \mathcal{L}_{\alpha}^{1}} g(\sqrt{|\lambda|} r)
$$


where $g(r)=f(r / \sqrt{|\lambda|})$. Let us start with a function $f \in L^{2}\left(\mathbb{R}_{+}, e^{\frac{1}{2}|\lambda| \xi^{2}} \xi^{2 \alpha+1}\right)$. Then

$$
\begin{aligned}
& |\lambda|^{-\alpha} \int_{\mathbb{C}}\left|e^{-i t \mathcal{L}_{\alpha}^{\lambda}} f(x+i y)\right|^{2} \theta_{|\lambda| t, \alpha}(\sqrt{|\lambda|} x, \sqrt{|\lambda|} y) d x d y \\
& =|\lambda|^{-\alpha-1} \int_{\mathbb{C}}\left|e^{-i t \mathcal{L}_{\alpha}^{\lambda}} f\left(\frac{x+i y}{\sqrt{|\lambda|}}\right)\right|^{2} \theta_{|\lambda| t, \alpha}(x, y) d x d y \\
& =|\lambda|^{-\alpha-1} \int_{\mathbb{C}}\left|e^{-i|\lambda| t \mathcal{L}_{\alpha}^{1}} g(x+i y)\right|^{2} \theta_{|\lambda| t, \alpha}(x, y) d x d y \\
& =|\lambda|^{-\alpha-1} \int_{0}^{\infty}|g(\xi)|^{2} e^{\frac{\xi^{2}}{2}} \xi^{2 \alpha+1} d \xi \\
& =|\lambda|^{-\alpha-1} \int_{0}^{\infty}|f(\xi / \sqrt{|\lambda|})|^{2} e^{\frac{\xi^{2}}{2}} \xi^{2 \alpha+1} d \xi \\
& =\int_{0}^{\infty}|f(\xi)|^{2} e^{\frac{|\lambda|}{2} \xi^{2}} \xi^{2 \alpha+1} d \xi .
\end{aligned}
$$

The uniqueness of weight function follows from the fact that the weight in Theorem 4.1 is unique. We summarize the above discussion in the following theorem.

Theorem 4.6 For any $t$ with $|\lambda t| \neq m \pi, e^{-i t \mathcal{L}_{\alpha}^{\lambda}}$ defines an onto isomorphism between $L^{2}\left(\mathbb{R}_{+}, e^{\frac{|\lambda|}{2} \xi^{2}} \xi^{2 \alpha+1}\right)$ and the Hilbert space of even entire functions on $\mathbb{C}$ which are square integrable with respect to the weight $\theta_{t, \alpha}^{\lambda}(x, y)=|\lambda|^{-\alpha} \theta_{|\lambda| t, \alpha}(\sqrt{|\lambda|} x, \sqrt{|\lambda|} y)$ and moreover the weight function is unique.

The solution of the Schrödinger equation associated with the operator $\mathcal{L}_{\alpha}$ with initial condition $f$ is given by

$$
e^{-i t \mathcal{L}_{\alpha}} f(x, \xi)=\int_{\mathbb{R}} e^{-i \lambda \xi} e^{-i t \mathcal{L}_{\alpha}^{\lambda}} f^{\lambda}(x) d \lambda .
$$

From the relation $e^{-t \mathcal{L}_{\alpha}^{\lambda}} f^{\lambda}(x)=e^{-t|\lambda| \mathcal{L}_{\alpha}^{1}} g(\sqrt{|\lambda|} x)$, where $g(x)=f^{\lambda}(x / \sqrt{|\lambda|})$ we deduce that

$$
\begin{aligned}
& e^{-i t \mathcal{L}_{\alpha}} f(x, \xi) \\
& =\int_{\mathbb{R}} e^{-i \lambda \xi} \int_{0}^{\infty}|\lambda|^{\alpha+1} K_{i|\lambda| t}(\sqrt{|\lambda|} x, \sqrt{|\lambda|} r) f(r) r^{2 \alpha+1} d r d \lambda .
\end{aligned}
$$


Let us note that

$$
|\lambda|^{\alpha+1} K_{i|\lambda| t}(\sqrt{|\lambda|}(x, r))=c_{\alpha} \frac{\lambda}{\sin (\lambda t)} e^{-\frac{i}{4} \lambda \cot (\lambda t)\left(x^{2}+r^{2}\right)} \frac{J_{\alpha}\left(\frac{\lambda x r}{\sin \lambda t}\right)}{(x r)^{\alpha}}
$$

has singularities at the points where $\lambda t$ is an integral multiple of $\pi$. But there is no singularity at $\lambda=0$. Therefore, if we assume that $\lambda \rightarrow f^{\lambda}$ is supported in $|\lambda| \leq R$, then the above integral operator makes sense as long as $|t|<\frac{\pi}{R}$. For each $R>0$ we introduce the space $\mathcal{G}_{R}$ a Hilbert subspace of $L^{2}\left((0, \infty) \times \mathbb{R}, r^{2 \alpha+1} d r d \xi\right)$ consisting of functions $f$ having the following properties: (i) $f^{\lambda}$ is supported in $|\lambda| \leq R$ and (ii) $\int_{\mathbb{R}} \int_{0}^{\infty}\left|f^{\lambda}(r)\right|^{2} e^{\frac{|\lambda|}{2} r^{2}} r^{2 \alpha+1} d r d \lambda$. We define $\mathcal{G}_{t}^{\lambda}$ as the space of all even entire functions $F$ for which

$$
\int_{\mathbb{C}}|F(x+i y)|^{2} \theta_{t, \alpha}^{\lambda}(x, y) d x d y<\infty .
$$

Then the following theorem characterizes the image of $\mathcal{G}_{R}$ under $e^{i t \mathcal{L}_{\alpha}}$ as a direct integral.

Theorem 4.7 Let $R>0$ and $|t|<\frac{\pi}{R}$. Then $e^{i t \mathcal{L}_{\alpha}}: \mathcal{G}_{R} \rightarrow \int_{-R}^{R} \oplus \mathcal{G}^{\lambda}{ }_{t} d \lambda$ is (up to a constant multiple) an isometric isomorphism.

Proof As we know that $\left(e^{i t \mathcal{L}} f\right)^{\lambda}=e^{i t \mathcal{L}_{\alpha}^{\lambda}} f^{\lambda}$ the theorem is proved by using the Euclidean Plancherel formula in the last variable and Theorem 4.6.

Now we take up the problem of characterizing the image of $\mathcal{G}_{R}$ under $e^{-i t \mathcal{L}_{\alpha}}$ as a weighted Bergman space. We note that the space $\mathcal{G}_{R}$ is invariant under translation in the last variable. Therefore, arguing as in Theorem 3.1 of the previous section we conclude that if there exists a nonnegative weight function $\Theta_{t, \alpha}(x+i y, \xi+i \eta)$ on $\mathbb{C} \times \mathbb{C}$ such that

$$
\begin{aligned}
& \int_{\mathbb{C}^{2}}\left|e^{i t \mathcal{L}_{\alpha}} f(x+i y, \xi+i \eta)\right|^{2} \Theta_{t, \alpha}(x+i y, \xi+i \eta) d x d y d \xi d \eta \\
& =c_{\alpha} \int_{\mathbb{R}} \int_{0}^{\infty}\left|f^{\lambda}(r)\right|^{2} e^{\frac{|\lambda|}{2} r^{2}} r^{2 \alpha+1} d r d \lambda
\end{aligned}
$$

then $\Theta_{t, \alpha}(x+i y, \xi+i \eta)=\Theta_{t, \alpha}(x+i y, i \eta)$ and satisfies the following identity:

$$
\int_{-\infty}^{\infty} e^{2 \lambda \eta} \Theta_{t, \alpha}(x+i y, i \eta) d \eta=c_{\alpha} \theta_{t, \alpha}^{\lambda}(x, y)
$$

for $|\lambda| \leq R$. 
Let us look into the weight function $\theta_{t, \alpha}^{\lambda}(x, y)$ more closely. We know that

$$
\begin{aligned}
& \theta_{t, \alpha}^{\lambda}(x, y) \\
& \quad=|\lambda|^{-\alpha} \theta_{|\lambda| t, \alpha}(x, y) \\
& \quad=|\lambda|^{-\alpha}(2 \sin |\lambda| t)^{2 \alpha} e^{\lambda(2 \cot (\lambda t)-\csc (\lambda t) x y)} h_{\sin ^{2}(|\lambda| t), \alpha}(\sqrt{|\lambda|}(x, y)) .
\end{aligned}
$$

We define for $\lambda \geq 0$

$$
\begin{aligned}
\theta_{t, \alpha}^{\lambda,+}(x, y) \\
=c_{\alpha}\left(x^{2}+y^{2}\right)^{-\frac{1}{2}} \lambda^{\alpha+\frac{1}{2}}\left(\frac{\sin (\lambda t)}{\lambda}\right)^{2\left(\alpha+\frac{1}{2}\right)} e^{-\left(\frac{\lambda}{\sin (\lambda t)}\right)^{2} \frac{1}{\lambda} y^{2}} \\
\quad \times \int_{0}^{\infty} e^{-\xi} \xi^{\alpha-\frac{1}{2}}\left(1+\frac{\xi}{2}\left(\frac{\sin (\lambda t)}{\lambda}\right)^{2} \frac{\lambda}{x^{2}+y^{2}}\right)^{\alpha-\frac{1}{2}} d \xi
\end{aligned}
$$

and for $\lambda<0$

$$
\begin{aligned}
\theta_{t, \alpha}^{\lambda,-}(x, y) & \\
= & c_{\alpha}\left(x^{2}+y^{2}\right)^{-\frac{1}{2}}(-\lambda)^{\alpha+\frac{1}{2}}\left(\frac{\sin (\lambda t)}{\lambda}\right)^{2\left(\alpha+\frac{1}{2}\right)} e^{\left(\frac{\lambda}{\sin (\lambda t)}\right)^{2} \frac{1}{\lambda} y^{2}} \\
& \times \int_{0}^{\infty} e^{-\xi} \xi^{\alpha-\frac{1}{2}}\left(1-\frac{\xi}{2}\left(\frac{\sin (\lambda t)}{\lambda}\right)^{2} \frac{\lambda}{x^{2}+y^{2}}\right)^{\alpha-\frac{1}{2}} d \xi .
\end{aligned}
$$

Writing down $\theta_{t, \alpha}^{\lambda}$ separately for $\lambda>0$ and $\lambda<0$ we have

$$
\theta_{t, \alpha}^{\lambda}=\left\{\begin{array}{l}
\theta_{t, \alpha}^{\lambda,+} \text { if } \lambda \geq 0 \\
\theta_{t, \alpha}^{\lambda,-} \text { if } \lambda<0 .
\end{array}\right.
$$

We note that $\theta_{t, \alpha}^{\lambda,+}, \theta_{t, \alpha}^{\lambda,-}$ have holomorphic extension to the right and left half planes respectively. Though the function $\theta_{t, \alpha}^{\lambda}(x, y)$ is continuous at $\lambda=0$ it is not analytic at $\lambda=0$. From the discussion in the previous section this observation is sufficient to conclude that there does not exist any non negative weight function $\Theta_{t, \alpha}(x+i y, \xi+i \eta)$ such that

$$
\begin{aligned}
& \int_{\mathbb{C}^{2}}\left|e^{-i t \mathcal{L}_{\alpha}} f(x+i y, \xi+i \eta)\right|^{2} \Theta_{t, \alpha}(x+i y, \xi+i \eta) d x d y d \xi d \eta \\
& =c_{\alpha} \int_{\mathbb{R}} \int_{0}^{\infty}\left|f^{\lambda}(r)\right|^{2} e^{\frac{|\lambda|}{2} r^{2}} r^{2 \alpha+1} d r d \lambda .
\end{aligned}
$$


Acknowledgments Part of this work was done while the third author was visiting Allahabad. He wishes to thank Harish-Chandra Research Institute for the hospitality during his stay. His work is also supported in part by J.C. Bose Fellowship from D.S.T. The authors wish to thank the referee for his/her careful reading of the manuscript and for making very useful suggestions that have been incorporated in the revised version.

\section{References}

1. Bargmann, V.: On a Hilbert space of analytic functions and an associated integral transform. Commun. Pure Appl. Math. 14, 187-214 (1961)

2. Cholewinski, F.M.: Generalised Fock spaces and associated operators. SIAM J. Math. 15, 177202 (1984)

3. Erdelyi, A. et al.: Higher Transcendental Functions, vol. 2. McGraw-Hill, New York (1953)

4. Folland, G.B.: Harmonic Analysis in Phase Space. Annals of Math Studies, vol. 122. Princeton University Press, Princeton (1989)

5. Folland, G.B.: Real Analysis, Modern Techniques and Their Applications. Wiley-Interscience, New York (1984)

6. Furioli, G., Veneruso, A.: Strichartz inequalities for the Schrödinger equation with the full Laplacian on the Heisenberg group. Studia Math. 160(2), 157-178 (2004)

7. Garg, R., Thangavelu, S.: On the Hermite expansions of functions from the Hardy class. Studia Math. 198(2), 177195 (2010)

8. Goldberg, M., Schlag, W.: Dispersive estimates for Schrödinger operator in dimension one and three. Commun. Math. Phys. 251(1), 157-178 (2004)

9. Hayashi, N., Saitoh, S.: Analyticity and smoothing effect of Schrödinger equation. Ann. Inst. Henri Poincare 52(2), 163-173 (1990)

10. Jenson, A.: Commutator methods and a smoothing property of the Schrödinger evolution group. Mathe. Z. 191, 53-59 (1986)

11. Journé, J.L., Soffer, A., Sogge, C.D.: Decay estimates for Schrödinger operators. Commun. Pure Appl. Math. XLIV, 573-604 (1991)

12. Karp, D.: Square summability with geometric weights for classical orthogonal expansions. In: Begehr, H.G.W., et al. (eds.) Advances in Analysis, pp. 407-422. World Scientific, Singapore (2005)

13. Keel, M., Tao, T.: End point Strichartz estimates. Am. J. Math. 120, 955-980 (1998)

14. Krötz, B., Thangavelu, S., Xu, Y.: The heat kernel transform for the Heisenberg group. J. Funct. Anal. 225, 301-336 (2005)

15. Lebedev, N.N.: Special Functions and Their Applications. Dover, New York (1992)

16. Nandakumaran, A.K., Ratnakumar, P.K.: Schrödinger equation and the regularity of the oscillatory semigroup for the Hermite operator. J. Funct. Anal 224, 371-385 (2005)

17. Nandakumaran, A.K., Ratnakumar, P.K.: Corrigendum Schrödinger equation and the regularity of the oscillatory semigroup for the Hermite operator. J. Funct. Anal. 224, 719-720 (2006)

18. Ratnakumar, P.K.: On Schrödinger propogator for the special Hermite operator. J. Fourier Anal. Appl. 14(2), 286-300 (2008)

19. Rudin, W.: Principle of Mathematical Analysis. McGraw-Hill International Editions, New York (1976)

20. Shohat, J., Tamarkin, J.: The Problem of Moments. American Math. Soc., Mathematical Surveys, vol. II. AMS, New York (1943)

21. Strichartz, R.S.: Restrictions of Fourier transforms to quadratic surfaces and decay of solutions of wave equations. Duke Math. J. 44(3), 705-714 (1977)

22. Strichartz, R.S.: Harmonic analysis as spectral theory of Laplacians. J. Funct. Anal. 87, 51-148 (1989)

23. Stempak, K.: On connections between Hankel, Laguerre and Jacobi transplantations. Tohoku Math. J. (2) 54(4), 471-493 (2002)

24. Stempak, K., Trebels, W.: On weighted transplantation and multipliers for Laguerre expansions. Math. Ann. 300, 203-219 (1994)

25. Thangavelu, S.: Lectures on Hermite and Laguerre Expansions, Mathematical Notes, vol. 42. Princeton University Press, Princeton (1993)

26. Thangavelu, S.: Harmonic Analysis on the Heisenberg Group. Progress in Math., vol. 154. Birkhäuser, Boston (1998)

27. Thangavelu, S.: Hermite and Laguerre semigroups: some recent developments. Séminaires et Congrès 25, 251-284 (2012) 